\title{
Performance Evaluation of FDL Effect in the Optical Switch
}

\author{
Jih-Hsin Ho \\ Department of Leisure \& Information Management, Taiwan Shoufu University, No.168,Nanshi Li, Madou Dist., Tainan City, \\ Taiwan.
}

\begin{abstract}
This paper studies the length effect of FDL (fiber delay line) in the optical switch nodes of CSMA/CP Metro ring network that is an all-optical WDM network for supporting variable packet transmission. This research takes into consideration the effect of guard-band distance between packets by simulations over a broad range of parameters. From the results, bandwidth efficiency and access delay of the network system have been influenced in evidence under different channels and guard-band distance. This paper provides a practical asymptote of superior FDL in optical switching node of CSMA/CP networks to reach higher throughput and lower delay.
\end{abstract}

Key words: Fiber Delay Line, CSMA/CP, Simulation.

\section{Introduction}

With the explosion of information traffic due to the Internet, electronic commerce, computer networks, voice, data and video, the need for a transmission medium with the bandwidth capabilities for handling such a vast amount of information is paramount. Recently, the channel bandwidth of commercial WDM (wavelength division multiplexing) communication systems has reached to OC-192 (10 Gbps) channels in one single fiber, and the total bandwidth of an optical fiber exceeds 1 Tbps. In order to efficiently use the bandwidth at WDM layer, it requires to develop the corresponding frameworks and protocols at higher layers. Hornet, IEEE 802.17 RPR, KOMNET, et al. developed as a testbed for a packet over WDM ring MAN [1-4] Today, optical switching can be divided into three paradigms: OCS (optical circuits switching), OBS (optical burst switching) and OPS (optical packet switching). OCS is a coarse switching. Because the number of the useable wavelength is constrained by the performance of the

Corresponding author: Jih-Hsin Ho, Ph.D., assistant professor, research fields: performance evaluation, WDM networks, Internet, QoS. E-mail: hjsin@tsu.edu.tw. devices, wavelength routing is not the ideal switching paradigm to realize all optical networks. The OPS enables routing of ultrahigh bit-rate packets in the optical layer and is a key technology for next generation. Therefore, in this paper focus on OPS topic. In order to support OPS WDM ring networks and satisfy the all optical communications and spatial reuse requirements, we had investigated an optical switching node of CSMA/CP [1] networks. The asynchronous variable length packet approach definitely matches better with IP traffic [5]. Due to the lack of optical random access memory, optical FDL (fiber delay line) is currently the only way to implement optical buffering. Feed forward and feedback are two kinds of FDL structures in optical buffering. Both have advantages and disadvantages. In this paper, we propose a feed-forward effective FDL architecture in optical switching node of Metro ring network. This paper studies superior length FDL in taking into consideration the effect of guard-band distance between packets. The guard-band distance is formed with switching time of SOA (semiconductor optical amplifier) and wavelength time of tunable transmitter. This paper studies superior length FDL in 

replicated simulations using different seeds. The parameters of the simulation are listed in Table 1 . The network throughput is calculated by the sum of total traffic that left all nodes in the ring network.

The simulation results presented in Fig. 2 and Fig. 3 show that the throughput per node of different $\mathrm{Tg}$ values under fixed load by increasing the FDL lengths. According to the simulation's results, we can find the appropriate length of FDL in the fourth channels and the eighth channels is approaching 140 bytes. Figs. 4 and 5 illustrate the simulation results for the transmission delay of the different $\mathrm{Tg}$ values by increasing the FDL lengths. They show that the simulation has a lower transmission delay, when FDL length is about 140 bytes. Thus, the appropriate length of FDL is equal to 140 bytes.

Table 1 Network parameters.

Architecture : TT-FR ${ }^{\mathrm{W}}$

Number of Nodes $(N) \quad 16$ (Fixed)

Number of Channels $(W) \quad 4,8$

Light Velocity $2 \times 10^{8} \mathrm{~m} / \mathrm{s}$

Ring Network Length $100 \mathrm{~km}$

Channel Speed $10 \mathrm{~Gb} / \mathrm{s}$ (OC-192)

Guard-time Distance $\left(\mathrm{T}_{\mathrm{g}}\right)$ 5, 12.5, 40, 48, and 64 Bytes

FDL Length $(L) \quad(40+\mathrm{Tg}) \sim(1500+\mathrm{Tg})$ Bytes

Packet Range OC-3 Traffic Distribution and Poisson Process

Average Packets Length 353 Bytes
Fig. 3 Comparing throughput with Tg length in channels under load=3.63 Gb/s.

Fig. 4 Comparing transmission delay with Tg length in 4 channels under Load=6.89 Gb/s.

Fig. 5 Comparing transmission delay with Tg length in 8 channels under load=3.63 Gb/s.
Fig 2 Comparing throughput with Tg length in 4 channels under load=6.89 Gb/s. 
